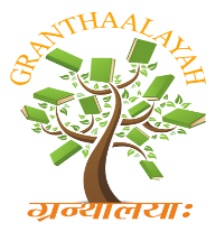

\author{
INTERNATIONAL JOURNAL OF RESEARCH - \\ GRANTHAALAYAH \\ A knowledge Repository
}

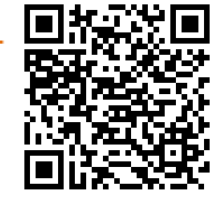

\title{
ECO-FRIENDLY TECHNOLOGY-KEY FOR SUSTAINABLE DEVELOPMENT
}

\author{
Sarika Tundele \\ Govt. College, Barwaha, District-Khargone (M.P.)
}

\begin{abstract}
Environmentally friendly technology is a rapidly growing field that focuses on new scientific and technical methods that benefit the earth. Also referred to as "green technology," this area is dedicated to preserving our natural resources. This involves both the development of new technologies and the improvement of existing ones. While these inventions, improvements, and scientific developments can have a worldwide impact on our global ecosystem, they also affect people on an individual level. Many green technologies directly affect the everyday lives of those who use them. By improving the way people perform basic tasks such as cooking, cleaning, or heating and cooling their home, green technologies are able to reduce the environmental impact that families have on the earth every day. Environmentally friendly technology utilizes many methods for reducing the impact that various activities have upon the earth. To be considered environmentally friendly, a product or action should be sustainable, produce as little waste and pollution as possible, and utilize the recycling and reuse of materials whenever possible.

\section{Keywords:}

Eco-friendly technology, green technology, sustainable, Renewable, styrofoam and polyethylene, biodegradable, green building.
\end{abstract}

\section{INTRODUCTION}

Eco-friendly technology is also known as clean tech, green tech and environmental tech, ecofriendly technology can help preserve the environment through energy efficiency and reduction of harmful waste. Green tech innovators use the latest environmental science and green chemistry to reduce the harmful impact of human activity on the earth.

Green technology is still in the earliest stages of development, but many exciting innovations have already been made in areas like renewable energy, water purification and waste management, as well as in everyday consumer products like electronics and vehicles. It can be as small as a handheld gadget or as expansive as a new method of filtering greenhouse gases out of the atmosphere.

\section{METHODOLOGY}

- Eco-friendly technology often involves some of the following:

- Recycled, recyclable and/or biodegradable content

- Plant-based materials

- Reduction of polluting substances 
- Reduction of greenhouse gas emissions

- Renewable energy

- Energy-efficiency

- Multi-functionality

- Low-impact manufacturing

Energy is one well-known area of green technology. Sustainable sources of energy include wind power, hydroelectric power, biofuels, and solar energy. These environmentally friendly technologies can now be used to power homes, businesses, and even small electronic devices. Solar-powered garden lights, remote controls, and electric shavers are all available for ecoconscious consumers.

Recently, new innovations in the field of green technology have included highly efficient LED lights, which could be used to make countless other gadgets more environmentally friendly, as well as promising growth in lesser-known renewable energies like algae oil. A company called Agilyx is working on technology that could turn plastic trash into synthetic crude oil, and flexible thin-film solar modules are one step closer to making a wider range of solar energy applications possible.

Major companies like Dell and Google are taking notable steps forward with eco-friendly technology in projects ranging from plant-based packaging to vast wind farms. Google has announced that it will invest \$100 million in the Shepherd's Flat wind project in Oregon, which will supply an average of 235,000 homes once it's fully operational in 2012 . The tech-driven company is particularly interested in the project because it will be the first to use directdrive turbines supplied by GE. But wind power isn't the only renewable energy Google has its eye on; the company announced in early April 2011 that it will invest \$168 million in a utility-scale power plant in the California desert, and also bought a 49-percent stake in a photovoltaic farm in Germany. Such investments could help the tech giant power its own energy-hungry operations more sustainably. Google currently has a 1.6 megawatt solar installation of its own at its Mountain View, California headquarters. Dell is bringing its latest eco-friendly technology closer to home - specifically, the homes of customers ordering the company's computers. Dell has announced a new sustainable packing strategy that will use mushrooms to create product cushioning for shipment. Grown rather than manufactured, the mushroom-based packaging is produced when agricultural waste products like cotton hulls are pressed into molds and then inoculated with mushroom spawn. Within five to ten days, the resulting packaging is ready to use. Mushroom-based packaging is biodegradable, making it a far greener option than commonly used styrofoam and polyethylene.

Some environmentally friendly technologies focus on everyday consumer products such as cleaning supplies, paint, and plastics. An increasing number of companies such as method® and Seventh Generation ${ }^{\circledR}$ have emerged to provide consumers with cleaning products that are safe for the earth. Even leading global companies such as Procter \& Gamble ${ }^{2}$, which produces Dawn $®$, Tide $®$, Gain ${ }^{\circledR}$, Bounty®, Puffs $®$, Charmin $®$, and other well-known brands, have gotten on board, setting goals for sustainability.

On a larger scale, green building has emerged as an important area of environmentally friendly technology. Green buildings use sustainable or recycled materials as much as they can and strive to impact the surrounding environment as little as possible. These structures often include innovative features such as solar panels for clean energy and energy efficient appliances that use less power and water. 


\section{CONCLUSION}

As awareness of our impact on the environment increases, environmentally friendly technology will likely expand as well. The future of this field may see innovations we can't yet begin to fathom as society strives to live lightly upon the earth and reduce and repair the damaging impacts of our ever-increasing population. 